\title{
Tako Tsubo Cardiomyopathy Secondary to Seizures
}

\author{
S.G. Weeks, N. Alvarez, N. Pillay, R.B. Bell
}

Can. J. Neurol. Sci. 2007; 34: 105-107

Tako Tsubo cardiomyopathy was first described in Japan in 1990 by Satoh et al. ${ }^{1}$ This syndrome of transient left ventricular dysfunction in response to severe emotional stress or illness occurs in the setting of normal epicardial coronary arteries. Patients are most commonly females without prior history of cardiac disease. The syndrome consists of chest pain, EKG abnormalities and elevation of cardiac markers. The resulting left ventricular dysfunction primarily affects the anterior and apical segments, creating an image of apical ballooning on left ventriculogram. The term Tako Tsubo is Japanese for octopus trap, which the left ventriculogram appearance resembles. This letter highlights presentation of Tako Tsubo cardiomyopathy secondary to a seizure.

A 64-year-old right-handed female was brought to the emergency department after being found experiencing a generalized tonic-clonic seizure. Significant medical history included: seizure disorder of undetermined etiology since age nine, current smoker, hypertension, depression, possible asthma, and bilateral mastectomies for fibrosystic breast diease. The day prior to admission, the patient described a vague sensation of being "unwell". The seizure leading to admission was of unknown duration but felt to be typical of her prior events.

The patient received $2.5 \mathrm{mg}$ of midazolam from paramedics with seizure resolution. However, she experienced a subsequent seizure in the emergency department. Initial vital signs were: BP 160/66, HR 116, RR 18, 100\% oxygen saturation on 3L by nasal drip. The patient appeared dyspneic to emergency staff. Initial EKG was abnormal with minor ST elevation in leads V2 and V3 (Figure 1). Chest x-ray revealed pulmonary edema. An echocardiogram was performed in the emergency department confirming antero-lateral papillary muscle dysfunction, mild mitral regurgitation, and slightly dilated left ventricle with moderate-severe systolic dysfunction secondary to antero-apical akinesis (Figure 2). Cardiac markers were elevated (Troponin T peak $1.54 \mu \mathrm{g} / \mathrm{L})$. A CT head scan revealed a remote left caudate lacunar infarct. Carbamazepine level was in the low therapeutic range (20-50 umol/L) at 23.9.

The patient was admitted to the coronary care unit for management of suspected acute coronary syndrome and congestive heart failure. Her initial therapy included: ASA, atorvastatin, topical nitrates, IV heparin, ace-inhibitor, phenytoin and carbamazepine. She improved clinically and underwent cardiac catheterization within a week of presentation. Left ventricular end-diastolic pressure was $14 \mathrm{~mm} \mathrm{Hg}$ with severe anterolateral and apical systolic impairment. Ejection fraction

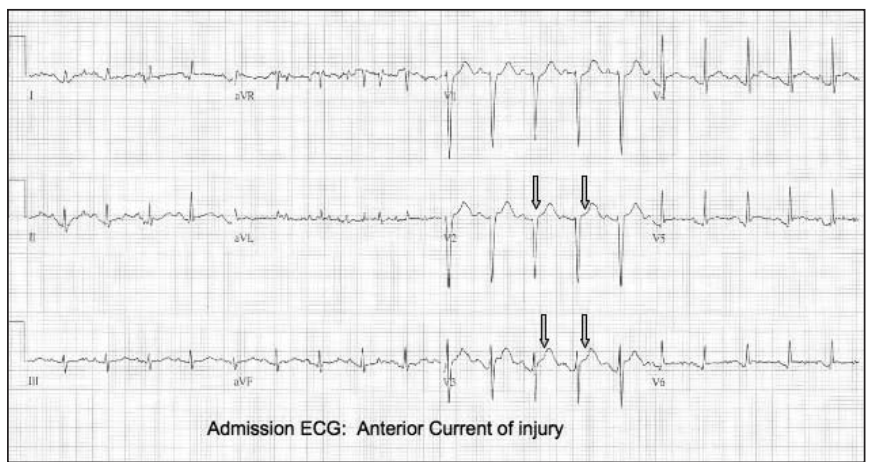

Figure 1A: Admission ECG reveals anterior injury pattern with $S T$ elevation in leads V2 and V3 (see arrows).

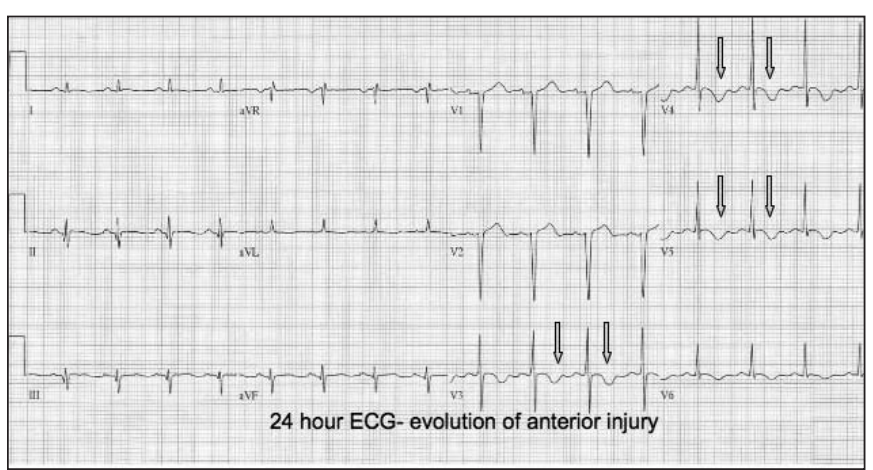

Figure 1B: ECG at 24 hours reveals evolution of anterior injury pattern with T wave inversions lead V3-V5 (see arrows).

From the Department of Clinical Neurosciences, University of Calgary, Health Sciences Centre, Calgary, Alberta, Canada.

ReCEIVED MAY 26, 2006. ACCEPTED IN FINAL FORM NOVEMBER 23, 2006. Reprint requests to: Robert B. Bell, Department of Clinical Neurosciences, University of Calgary, Health Sciences Centre, 3330 Hospital Drive NW, Calgary, Alberta, T2N 4N1, Canada. 


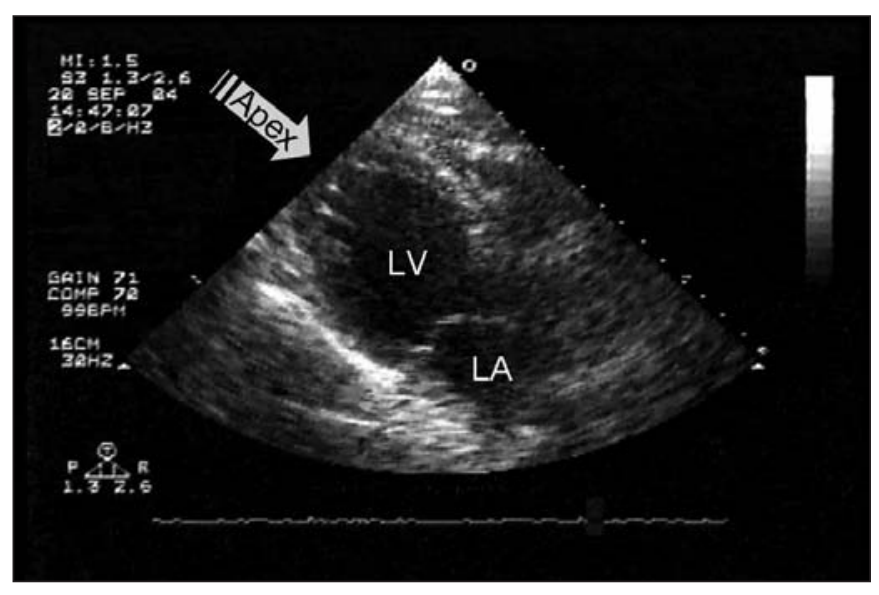

Figure 2A: Echocardiogram on admission: apical three chamber view in diastole reveals a spherical apex $(L A=$ left atrium, $L V=$ left ventricle $)$.

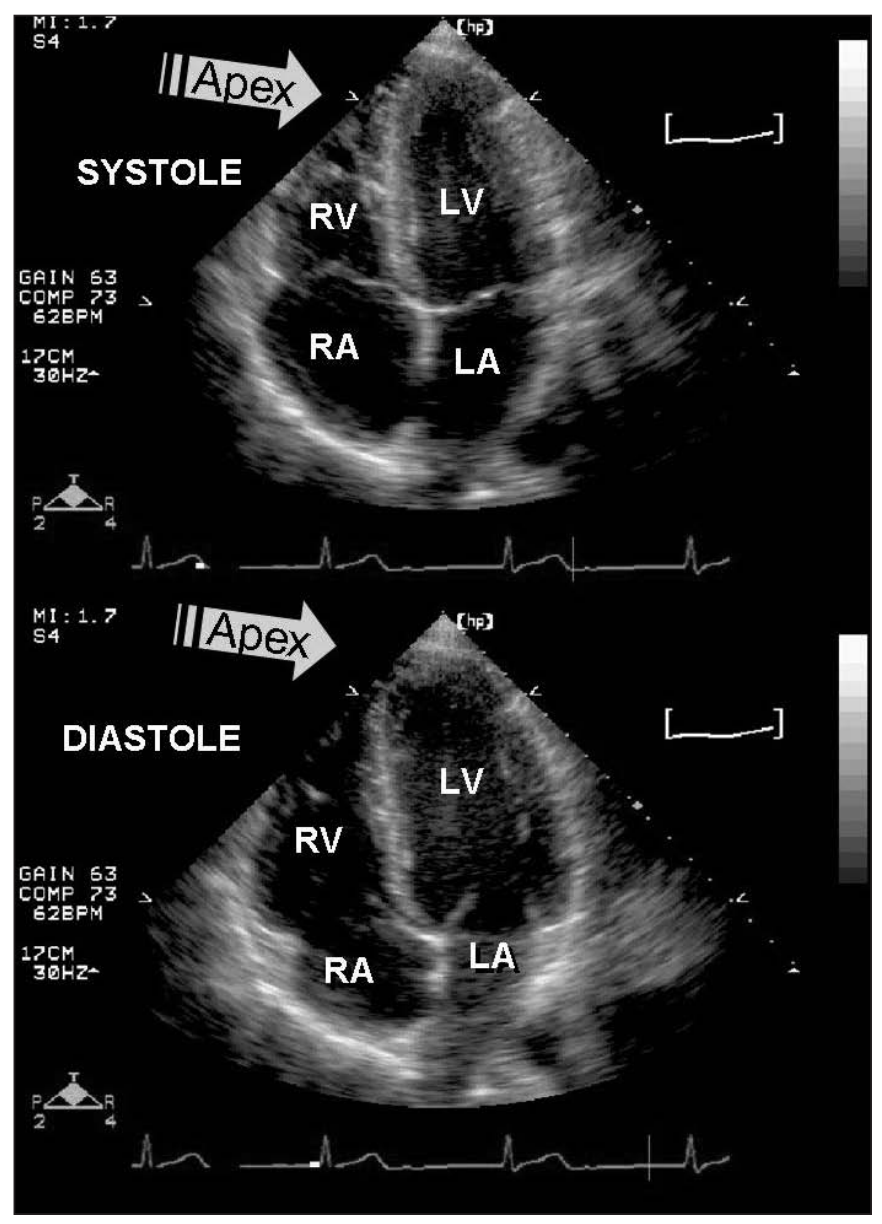

Figure 2B: Systole: right anterior oblique projection of the left ventricle during systole with normal contraction of the basal segments (near the catheter tip) and "ballooning" of the apical segments. Diastole: right anterior oblique projection of the left ventricle during diastole with normal shape. was calculated at $25 \%$. There was no evidence of flow-limiting CAD, with one $10 \%$ lesion noted in the proximal right coronary artery (Figure 3). An EEG demonstrated generalized spikes, poly spikes maximal over the left temporal frontal region and supportive of a generalized seizure disorder.

Day nine of admission the patient was discharged home in stable condition on the following medications: ASA, carvedilol, losartan, clopidogrel, synthroid, paroxetine, atorvastatin, phenytoin and carbamazepine. An EKG on discharge had normalized. Following discharge, her anticonvulsant was changed to valproic acid.

Subsequent investigations including myocardial perfusion imaging and echocardiogram were completed at 12 and 14 weeks post discharge and confirmed the return of normal left ventricular systolic performance (ejection fraction 75\%).

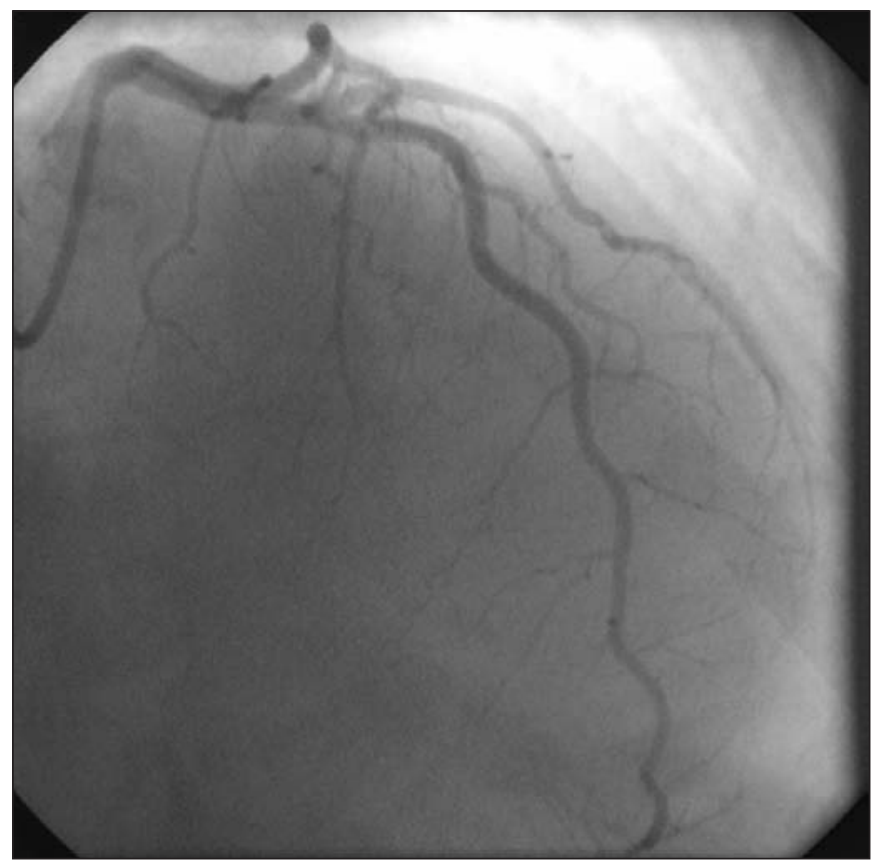

Figure 3A: Projection of the left anterior descending artery without evidence of obstructive coronary artery disease.

The first case series of Tako Tsubo in caucasians, by Desmet et $\mathrm{al}^{2}$ described 13 patients, $92 \%$ female with a mean age of 62 years. Nine patients had an identifiable triggering factor, none of which were seizure related. Chin et $\mathrm{al}^{3}$ have reported two cases of "stunned myocardium" subsequent to seizure activity.

The pathophysiology of Tako Tsubo is unclear. Typical myocardial infarction does not explain this syndrome given the lack of epicardial coronary artery disease, relatively minor elevation in cardiac markers for the extent of dysfunction and the dramatic recovery of systolic performance over a short period of 


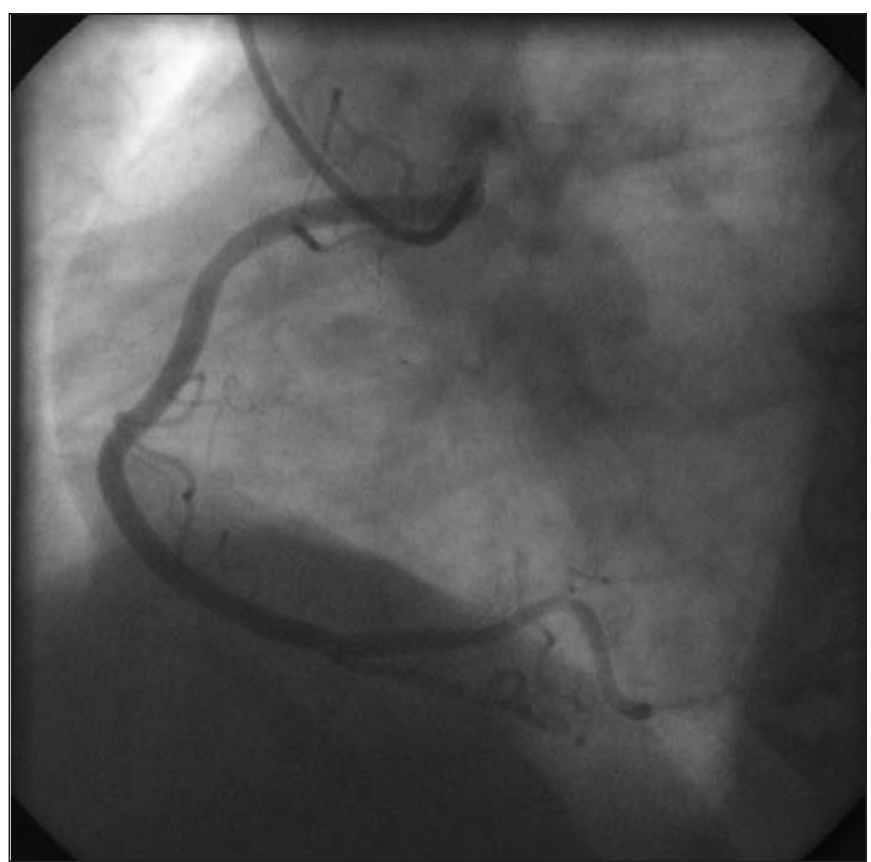

Figure 3B: Projection of the right coronary artery without evidence of obstructive coronary artery disease.

time. Multivessel coronary vasospasm has been theorized as the cause of myocardial stunning. However, only $21 \%$ of patients with this syndrome experienced coronary vasospasm under provocative testing. ${ }^{4}$

The consistent feature of Tako Tsubo cardiomyopathy is one of exposure to significant stress. Catecholamines and enhanced adrenergic stimulation are likely to play a pivotal role. Left ventricular outflow tract obstruction, similar to that seen in hypertrophic obstructive cardiomyopathy, has been demonstrated in three patients presenting with a similar syndrome. ${ }^{5}$ The proposed mechanism involves outflow obstruction, a rapid increase in left ventricular end-diastolic pressure and subsequent increased $\mathrm{O}_{2}$ demand with decreased myocardial perfusion. Unfortunately, this cannot be the sole cause as only $15 \%$ of the patients in case series by Desmet et al had a measurable intraventricular pressure gradient suggestive of outflow obstruction. ${ }^{2}$

Myocardial contraction bands with cell death have been reported in post mortem analyses of patients dying of status epileptcus. ${ }^{6}$ This pathological feature occurs in the myocardium adjacent to the insertion of the sympathetic end plates supporting the involvement of catecholamine release. The authors postulate that myocardial nerve terminal catecholamine release rather than systemic endogenously released catecholamines are responsible.

Myocardial injury has been demonstrated with other significant neurologic conditions. Subarachnoid hemorrhage frequently results in myocardial death..$^{7-10}$ The degree of neurologic injury is a strong independent predictor of myocardial necrosis. The pattern, however, tends to be one of global dysfunction, as opposed to localized anteroapical dysfunction, as is seen in Tako Tsubo. ${ }^{2}$
Tako Tsubo cardiomyopathy mimics acute myocardial infarction after exposure to physical or emotional stress. Physicians treating patients with seizures should be aware of this clinical entity. The pathophysiology has not been adequately elucidated. Patients are initially treated similarly to those with acute myocardial infarction at least initially. The transient nature of the systolic impairment confers positive outcomes as function returns to normal if the patient survives the initial insult.

\section{REFERENCES}

1. Satoh H, Tateishi H, Uchida T. [Takotsubo type cardiomyopathy due to multivessel spasm]. In: Kodama K, Haze K, Hon M, editors. [Clinical aspect of myocardial injury: from ischemia to heart failure] (in Japanese). Tokyo: Kagakuhyouronsya; 1990. p. $56-64$.

2. Desmet WJR, Adriaenssens BFM, Dens JAY. Apical ballooning of the left ventricle: first series in white patients. Heart. 2003;89: 1027-31.

3. Chin PS, Branch KR, Becker KJ. Postictal neurogenic stunned myocardium. Neurology. 2005;64:1977-8.

4. Tsuchihashi K, Ueshima K, Uchida T, Oh-mura N, Kimura K, Owa $\mathrm{M}$, et al. Transient left ventricular apical ballooning without coronary artery stenosis: a novel heart syndrome mimicking acute myocardial infarction. J Am Coll Cardiol. 2001;38(1): 11-18.

5. Villareal RP, Achari A, Wilansky S, Wilson JM. Anteroapical stunning and left ventricular outflow tract obstruction. Mayo Clin Proc. 2001;76(1):79-83.

6. Manno EM, Pfeifer EA, Cascino GD, Noe KH, Wijdicks EF. Cardiac pathology in status epilepticus. Ann Neurol. 2005;58(6):954-7.

7. Tung P, Kopelnik A, Banki N, Ong K, Ko N, Lawton MT, et al. Predictors of neurocardiogenic injury after subarachnoid hemorrhage. Stroke. 2004;35(2):548-51

8. Banki NM, Kopelnik A, Dae MW, Miss J, Tung P, Lawton MT, et al. Acute neurocardiogenic injury after subarachnoid hemorrhage. Circulation. 2005;112(21):3314-9.

9. Sato K, Masuda T, Izumi T. Subarachnoid hemorrhage and myocardial damage: clinical and experimental studies. Jpn Heart J. 1999;40:683-701.

10. Mayer S, Lin J, Homma S, Solomon R, Lennihan L, Sherman D, et al. Myocardial injury and left ventricular performance after subarachnoid hemorrhage. Stroke. 1999;30:780-6. 